\title{
Digital pleural drainage technology is here to stay-time to realize its potential
}

\author{
Ching Yeung ${ }^{1}$, Sebastien Gilbert ${ }^{1,2}$ \\ ${ }^{1}$ Division of Thoracic Surgery, University of Ottawa, Ottawa, Ontario, Canada; ${ }^{2}$ The Ottawa Hospital Research Institute, Ottawa, Ontario, Canada \\ Correspondence to: Sebastien Gilbert, MD, FRCSC. Division of Thoracic Surgery, University of Ottawa, The Ottawa Hospital, General Campus, \\ Suite 6363, 501 Smyth Road, Ottawa, Ontario K1H 8L6, Canada. Email: sgilbert@toh.ca. \\ Provenance: This is an invited Editorial commissioned by the Section Editor Shuangjiang Li (Department of Thoracic Surgery and West China \\ Medical Center, West China Hospital, Sichuan University, Chengdu, China). \\ Comment on: Takamochi K, Nojiri S, Oh S, et al. Comparison of digital and traditional thoracic drainage systems for postoperative chest tube \\ management after pulmonary resection: A prospective randomized trial. J Thorac Cardiovasc Surg 2018;155:1834-40.
}

Submitted Aug 03, 2018. Accepted for publication Sep 14, 2018.

doi: $10.21037 /$ jtd.2018.09.71

View this article at: http://dx.doi.org/10.21037/jtd.2018.09.71

Institutions worldwide seek means to continue to produce high quality care while reducing overall health care cost. Digital pleural drainage devices have been explored as a potential method to improve health care efficiency. Objective documentation of a parenchymal air leak using digital sensors results in increased inter-observer reliability $(1,2)$ thus presenting an opportunity to identify candidates for chest tube removal in a more reliable and timely fashion. With indwelling chest tubes often being a limiting factor in discharging patients from hospital, earlier removal may lead to improved length of stay.

In a single-center, prospective randomized trial of digital versus traditional pleural drainage after pulmonary resection, Dr. Takamochi and colleagues found no significant difference in the duration of chest tube drainage (median 2 vs. 3 days; $\mathrm{P}=0.149$ ) and length of hospital stay (median 6 vs. 7 days; $\mathrm{P}=0.584$ ) between groups (3). Both groups had similar incidences of prolonged air leak and adverse events. The investigators appropriately recognized that the results may be biased as the traditional device group had significantly more initial postoperative air leak than the digital group $(\mathrm{n}=44$ vs. $20 ; \mathrm{P}=0.012)$. Since air leak is a major determinant of the duration of chest drainage, it is reasonable to think that this difference may have affected the results. In a subgroup analysis performed on patients who had a postoperative air leak, the traditional device group had more clamping trials compared to the digital device group. We obtained similar findings during our own randomized trial of digital versus analog pleural drainage (1). It is also interesting to note that pleurodesis was used in a significantly higher proportion of patients randomized to digital pleural drainage. The decision to proceed with pleurodesis in patients with an air leak duration greater than or equal to 5 days was made according to the clinical judgment of the physician. This raises the question as to whether or not selection criteria for performing this bedside procedure was well established and agreed upon prior to initiating the trial.

There have been mixed results in previously published prospective randomized trials comparing digital and traditional pleural drainage devices (4-8). We concur that there are important methodological problems in previously published trials (4-6). These include, incomplete adherence to CONSORT guidelines, lack of control for postoperative air leak status, relatively small number of patients, and heterogeneity in postoperative chest tube management protocols. With the current state of the evidence, it seems reasonable to think that the use of digital pleural drainage devices may improve the duration of chest tube drainage. It may be argued that length of hospital stay is the only outcome that really matters. At the same time, length of stay can be affected by many other factors that are often unrelated to surgery and out of the control of the clinical team. Therefore, we think that length of stay may not be the best barometer by which to measure the effectiveness of new pleural drainage technology. 
Despite conflicting evidence about the benefits of digital devices, we agree with the authors' assessment that digital devices will eventually replace traditional drainage devices. The full potential of digital pleural drainage devices has yet to be realized. Currently, the devices are primarily used to determine presence or absence of an air leak. However, this monitoring technology brings a new stream of pleural space data that is waiting to be harnessed to streamline chest tube management. For instance, if we can develop accurate models of transpleural air flow in patients with an air leak, we may be better equipped to make timely decisions regarding interventions (e.g., trial of clamping, pleurodesis) and discharge with a portable pleural drainage device. By improving inter-observer reliability and providing a digital pleural drainage record, digital devices safely opens the door for nurses to take on a more preponderant role in providing chest tube care $(2,7)$. The continuous stream of pleural space data generated by these devices may also be amenable to analysis using machine learning algorithms in order to develop clinical decision-support systems.

Finally, we would like to congratulate the authors for their efforts and discipline in successfully contributing highlevel evidence to the care of patients with chest drains. We believe that, in the not so far future, digital drainage devices will become more commonplace items. The technology is definitely here to stay. The community of physicians and surgeons caring for patients with chest drains should continue to explore the relatively untapped potential of this technology.

\section{Acknowledgements}

We would like to thank Dr. Jennifer Dawson for her assistance in reviewing the manuscript.

\section{Footnote}

Conflicts of Interest: The authors have no conflicts of interest to declare.

\section{References}

1. Gilbert S, McGuire AL, Maghera S, et al. Randomized trial of digital versus analog pleural drainage in patients with or without a pulmonary air leak after lung resection. J Thorac Cardiovasc Surg 2015;150:1243-9.

2. McGuire AL, Petrcich W, Maziak DE, et al. Digital versus analogue pleural drainage phase 1: prospective evaluation of interobserver reliability in the assessment of pulmonary air leaks. Interact Cardiovasc Thorac Surg 2015;21:403-7.

3. Takamochi K, Nojiri S, Oh S, et al. Comparison of digital and traditional thoracic drainage systems for postoperative chest tube management after pulmonary resection: A prospective randomized trial. J Thorac Cardiovasc Surg 2018;155:1834-40.

4. Brunelli A, Cassivi SD, Salati M, et al. Digital measurements of air leak flow and intrapleural pressures in the immediate postoperative period predict risk of prolonged air leak after pulmonary lobectomy. Eur J Cardiothorac Surg 2011;39:584-8.

5. Cerfolio RJ, Bryant AS. The benefits of continuous and digital air leak assessment after elective pulmonary resection: a prospective study. Ann Thorac Surg 2008;86:396-401.

6. Filosso PL, Nigra VA, Lanza G, et al. Digital versus traditional air leak evaluation after elective pulmonary resection: a prospective and comparative monoinstitutional study. J Thorac Dis 2015;7:1719-24.

7. Lijkendijk M, Licht PB, Neckelmann K. Electronic versus traditional chest tube drainage following lobectomy: a randomized trial. Eur J Cardiothorac Surg 2015;48:893-8; discussion 898.

8. Pompili C, Detterbeck F, Papagiannopoulos K, et al. Multicenter international randomized comparison of objective and subjective outcomes between electronic and traditional chest drainage systems. Ann Thorac Surg 2014;98:490-6; discussion 496-7.
Cite this article as: Yeung C, Gilbert S. Digital pleural drainage technology is here to stay-time to realize its potential. J Thorac Dis 2018;10(Suppl 33):S3882-S3883. doi: 10.21037/jtd.2018.09.71 